\title{
La cobertura en la prensa aragonesa de dos campañas "nacionales": las elecciones europeas de 2009 frente a las generales de 2008
}

\section{The coverage of two 'national' campaigns in the Aragonese press: The 2009 European Elections compared to the 2008 General Elections}

\author{
Dr. Ricardo Zugasti Azagra \\ Profesor de la Facultad de \\ Ciencias de la Comunicación, \\ Universidad San Jorge (USJ) \\ rzugasti@usj.es
}

Dra. Carmela García Ortega

Profesora de la Facultad de Ciencias de la Comunicación, Universidad San Jorge (USJ)

cgarcia@usj.es

Recibido: 15 de enero de 2012 Aceptado: 31 de enero de 2012

\section{Resumen}

Este artículo demuestra, en la línea de otros trabajos, la consideración periodística de las elecciones europeas como elecciones de segundo orden en las que priman los componentes nacionales frente a los europeos. La novedad radica en que el estudio utiliza, por primera vez, una comparación con la cobertura de unas elecciones generales y que, además, se aborda en el contexto de la prensa regional. En concreto, se aplica un análisis de contenido cuantitativo a las coberturas de las campañas de las generales de 2008 y de las europeas de 2009 ofrecidas por los cuatro diarios de pago que se editan en Aragón.

\section{Abstract}

This article demonstrates, along the lines of other studies, the treatment by the press of the European elections as second-rate elections in which the national features outweigh the European focus. This piece of research uses for the first time a comparison with the coverage of the national elections in the context of the regional press. Specifically, a content analysis was applied to the coverage by the four main daily newspapers published in Aragon in relation to the 2008 general and the 2009 European election campaigns. 
Palabras Clave: periodismo; prensa; política.

Key Words: journalism; press; politics.

\section{Sumario}

1. Introducción. 2. Metodología. 3. Resultados. 4. Conclusiones. 5 Bibliografía. 6. Nota.

\section{Summary}

1. Introduction. 2. Methodology. 3. Results. 4. Conclusions. 5. Bibliography. 6. Notes.

\section{Introducción}

Son varios los estudios que, desde diferentes ámbitos y perspectivas, demuestran que los medios de comunicación cubren las elecciones europeas como comicios de segundo orden (Canel e Innerarity, 2000; Kevin, 2003; Maarek, 2007; Berganza, 2008a; Berganza, 2008b; Berganza, 2009; De Vreese, 2009). Es decir, los medios no plasman el interés de estas elecciones por sí mismas, sino que se entienden como un proceso electoral de segunda magnitud, a la sombra de otros considerados más importantes o decisivos por los partidos políticos y los propios votantes.

Este enfoque dominante explica los rasgos comunes de las coberturas periodísticas. En primer lugar, predominan los temas de política interna y los puntos de vista nacionales frente a los comunitarios. Siguiendo también la estrategia electoral diseñada por los propios partidos, lo prioritario durante este tipo de campañas no es mostrar cuestiones relacionadas directamente con Europa, puesto que las elecciones son vistas por los grupos políticos como una oportunidad para "medir sus niveles de popularidad" y comprobar su situación política interna (Berganza, 2008a: 17). En la cobertura se incide, además, en el enfrentamiento entre el partido en el poder y el principal grupo de la oposición. La inexistencia de un auténtico sistema de partidos europeo y de un único sistema electoral y, por tanto, de "una verdadera campaña europea" influye, según Morata, restringiendo en cierto modo la proyección de los partidos y, por consiguiente, también de sus temas y candidatos, y confinando sus campañas a un ámbito de actuación similar al de otros comicios de orden interno (2000: 23).

En segundo lugar, en la cobertura de las elecciones europeas hay un elevado grado de personalización concretada en los líderes de los partidos. Como es habitual en otras campañas electorales, "el mensaje electoral está representado en una persona concreta" que funciona como referente y con la que el votante puede identificarse más fácilmente (Canel, 2006: 48). Picarella señala, además, que "la personalización de las campañas electorales ha 
sido muy clara en todas las elecciones españolas, que se han concentrado, sin duda, sobre el líder" (2008: 6).

Sin embargo, en el caso de los comicios europeos esa propuesta electoral no se encarna en el candidato al Parlamento Europeo. Dado que los partidos consideran esta campaña como una especie de ensayo para medir sus fuerzas de cara a las elecciones generales, los líderes de esos grupos suelen tener una elevada visibilidad en los medios y eclipsan a los propios candidatos europeos (Berganza, 2008a: 27; Zurutuza Muñoz y García Ortega, 2010: 9-13).

Los diferentes actores políticos entienden, en definitiva, las elecciones al Parlamento Europeo como una vía para medir la popularidad de los partidos. La cobertura mediática, centrada sobre todo en cuestiones nacionales, que deriva de esa concepción, termina afectando al tipo de información y al conocimiento que la opinión pública tiene del Parlamento Europeo, una institución ya de por sí lejana y compleja, reforzando así lo que Galindo ha calificado como "divorcio de las instituciones europeas y sus ciudadanos" (2010: 69).

Los índices de participación en las elecciones del 7 de junio de 2009 en España confirman esta tendencia: el nivel de abstención superó a la movilización y alcanzó el 55,1\% del electorado. En cambio, en las últimas elecciones generales, celebradas el 9 de marzo de 2008, la participación fue del 73,8\%, unos 20 puntos porcentuales por encima. Además, la encuesta post-electoral del Centro de Investigaciones Sociológicas tras los comicios de junio de 2009 demuestra que el voto del 58,6\% de los encuestados estuvo influido principalmente por temas de política interna, y no por la Unión Europea (CIS, 2009).

El aporte diferenciador de este artículo radica, fundamentalmente, en que presenta por primera vez el análisis de una cobertura de campaña de elecciones europeas puesto en relación con el análisis de una cobertura de campaña de elecciones generales. De esta manera, es posible mostrar datos comparados acerca de las semejanzas y diferencias entre ambos tratamientos periodísticos. Además, es un estudio de la prensa diaria editada en la Comunidad Autónoma de Aragón, y no de los medios de comunicación de ámbito nacional, que es lo habitual en las investigaciones sobre comicios europeos realizadas hasta ahora. Todo ello permitirá profundizar en los rasgos de las coberturas electorales dando un paso más allá y comprobando si las constantes antes mostradas se producen también en el ámbito regional o si, por el contrario, existe un enfoque más localista que nacional.

Las coberturas que se describen y comparan en las próximas páginas son las realizadas por Heraldo de Aragón, El Periódico de Aragón, Diario del Altoaragón y Diario de Teruel durante los quince días anteriores a las últimas elecciones generales y europeas celebradas en España, el 9 de marzo de 2008 y el 7 de junio de 2009, respectivamente. La hipótesis de la que parte esta investigación es que las elecciones generales fueron cubiertas de manera 
más amplia por la prensa aragonesa que las europeas y que en estas últimas primó el encuadre nacional sobre el europeo o el aragonés. Estimamos asimismo que los candidatos en 2008, José Luis Rodríguez Zapatero y Mariano Rajoy, se convirtieron en protagonistas de la campaña electoral de 2009, por delante no sólo de los cabezas de lista de sus partidos, sino de otros candidatos, tanto de ámbito nacional como regional.

\section{Metodología}

La metodología empleada para llevar a cabo esta investigación es de corte cuantitativo: el análisis de contenido. Es ésta una técnica ampliamente utilizada en Ciencias Sociales, que se ha empleado con éxito en el campo de la Comunicación. Si bien la aplicación de este método a la Comunicación Política no es novedosa, sí lo es el ámbito en el que se desarrolla: la Comunidad Autónoma de Aragón. En este sentido, el presente trabajo resulta innovador y se encuentra entre los primeros de otros que se llevarán a término. Así pues, futuros estudios servirán para comprobar si las tendencias aquí apuntadas se consolidan o no.

Una de las principales ventajas del análisis de contenido es que permite realizar comparaciones a lo largo del tiempo, pues la realidad analizada se transforma en datos numéricos que se pueden poner en relación unos con otros: en este caso, los relativos a las elecciones generales de 2008 con los de las elecciones europeas de 2009.

Por tanto, este método de investigación sirve para realizar mediciones objetivas, válidas y fiables. Asimismo, permite describir de forma gráfica y sencilla la cobertura que los diarios seleccionados realizaron de ambas campañas electorales. En este sentido, es importante subrayar el carácter eminentemente descriptivo de esta investigación. No se pretende hacer inferencia, por lo que los resultados que se presentan a continuación sólo se deben aplicar a este caso concreto y no deberían extrapolarse a otros medios de comunicación o a otros periodos temporales.

Una última ventaja del análisis de contenido es que permite tratar un gran volumen de información. En este caso se trabaja con 2.163 unidades de análisis, una cifra muy elevada y difícil de manejar utilizando una metodología de corte cualitativo.

Esas unidades de análisis aparecieron publicadas en los cuatro diarios generalistas de pago de la Comunidad Autónoma de Aragón. Heraldo de Aragón, editado en Zaragoza, es la cabecera más importante de la Comunidad, con una difusión media de 53.087 ejemplares en 2008. Su principal competidor, El Periódico de Aragón, posee una difusión bastante menor (12.087 ejemplares). Por su parte, Diario del Altoaragón es la cabecera local de referencia en Huesca (con un promedio de difusión de 7.449 ejemplares) y Diario de Teruel, que no está sometido a los controles de la Oficina de Justificación de la Difusión (OJD), lo es en la tercera de las provincias aragonesas (AEDE, 2010: 46-47). 
Se definió como unidad de análisis cualquier información sobre las campañas y las elecciones de 2008 y 2009 que estuviera encabezada por un titular y gozara de entidad propia, independientemente del género en el que estuviera escrita. Es decir, se analizaron todo tipo de textos: noticias, reportajes, entrevistas, editoriales, artículos de opinión... Esta decisión encuentra su justificación en la Teoría del Framing, que afirma que los medios de comunicación encuadran la realidad sobre la que luego informan al público de acuerdo a factores muy diversos, es decir, que la información que los medios de difusión ofrecen a su audiencia responde al particular modo de acercarse a la realidad que tiene cada uno de ellos. Ese proceso de encuadramiento se produce, sobre todo, al seleccionar los acontecimientos que se convertirán en noticia $-\mathrm{y}$, consecuentemente, al dejar fuera otros- y al enfatizar determinados aspectos de un tema o dar un protagonismo especial a ciertos personajes (Sádaba, 2008). Por lo tanto, según esta teoría, los periódicos adoptaron una postura a la hora de informar sobre las elecciones generales y europeas y ésta no se puso de manifiesto sólo en los editoriales o en los artículos de opinión de los colaboradores habituales, sino en todo tipo de textos. De haber limitado este análisis únicamente a los géneros opinativos, habría quedado marginada una parte sustancial de escritos que, como afirma la Teoría del Framing, ampliamente desarrollada en los últimos años (Vicente Mariño y López Rabadán, 2009), también ofrece un determinado enfoque sobre la cuestión estudiada.

Las unidades de análisis fueron examinadas mediante el empleo de dos códigos que incluían variables formales y de contenido. Entre las primeras destacan, por ejemplo, la extensión del escrito, la autoría o el género, que ayudan a determinar la importancia que la prensa aragonesa otorgó a los textos relativos a las campañas electorales. Por lo que respecta al segundo tipo de variables, se atendió, de manera exhaustiva, a los temas y a los protagonistas principales de las noticias. Ambos códigos compartían una estructura común, para hacer posible la comparación, aunque los valores de algunas variables se adaptaron a características específicas de cada una de las elecciones estudiadas.

A continuación se presentan los resultados obtenidos tras el trabajo de campo y el análisis estadístico, que permitirán conocer cómo fueron las coberturas de las dos últimas campañas electorales en Heraldo de Aragón, El Periódico de Aragón, Diario del Altoaragón y Diario de Teruel.

\section{Resultados}

Durante la campaña de las elecciones generales de 2008, los cuatro periódicos objeto de estudio publicaron 1.561 unidades de análisis referidas a la campaña. El número de piezas periodísticas presentes en estos diarios durante este periodo fue 10.079, lo que supone que los artículos dedicados a las elecciones constituyeron casi el 15,5\% del total. Por otro lado, durante los quince días previos a las europeas de 2009, estos medios publicaron 602 escritos sobre la campaña, es decir, el 6,6\% de los 9.121 textos aparecidos en la prensa 
aragonesa durante esos días. Estos datos confirman la mayor importancia concedida por la prensa a las elecciones generales frente a las europeas.

La tabla 1 muestra cómo la distribución por cabeceras de las informaciones referidas a las elecciones fue bastante similar entre las generales y las europeas. En ambos casos destacan Heraldo de Aragón, como el medio que más artículos incluyó en sus páginas, y Diario de Teruel, como el que una menor cobertura dedicó a ambas campañas.

Tabla 1. Distribución de las unidades de análisis por diario

\begin{tabular}{|c|c|c|c|}
\hline Elecciones generales 2008 & $\%$ & Elecciones europeas 2009 & $\%$ \\
\hline Heraldo de Aragón & 33,4 & Heraldo de Aragón & 33,4 \\
\hline El Periódico de Aragón & 30,2 & El Periódico de Aragón & 25,4 \\
\hline Diario del Altoaragón & 21,3 & Diario del Altoaragón & 25,1 \\
\hline Diario de Teruel & 15,1 & Diario de Teruel & 16,1 \\
\hline Total & 100 & Total & 100 \\
\hline
\end{tabular}

Fuente: Elaboración propia

En cuanto a la extensión de los artículos publicados por el conjunto de la prensa aragonesa, al observar la tabla 2 se percibe una cierta semejanza entre las dos campañas electorales. La diferencia estriba en que en 2008 dominaron las piezas cortas frente a las medianas, que fueron las más habituales en $2009^{1}$.

Tabla 2. Extensión de la unidad de análisis

\begin{tabular}{|c|c|c|c|}
\hline Elecciones generales 2008 & $\%$ & Elecciones europeas 2009 & $\%$ \\
\hline Corta & 45,4 & Mediana & 38,2 \\
\hline Mediana & 29,3 & Corta & 33,2 \\
\hline Larga & 16,6 & Larga & 22,4 \\
\hline Muy larga & 8,6 & Muy larga & 6,1 \\
\hline Total & 100 & Total & 100 \\
\hline
\end{tabular}

Fuente: Elaboración propia

Por lo que respecta a la ubicación de las unidades de análisis, la tabla 3 muestra que la sección en la que se publicó un mayor número de artículos durante la campaña de 2008 fue la de Regional (30,1\%), frente a la de Nacional en la campaña de 2009 (35,5\%). En esta última, Regional fue la segunda sección que más noticias acaparó $(26,4 \%)$. Sumando los porcentajes referidos a Regional y Local se obtiene que el $40 \%$ de la cobertura de campaña

\footnotetext{
${ }^{1}$ Se consideraron como informaciones cortas las que no alcanzaban el cuarto de página; como medianas las que eran iguales o superiores a un cuarto de página, pero inferiores a media página; como largas las que ocupaban media página o más pero no llegaban a los tres cuartos; y como muy largas las que sobrepasaban los tres cuartos de página.
} 
en las elecciones generales se concentró en estas secciones. Similar es el dato, 38,4\%, obtenido en la campaña de las europeas. El enfoque aragonés de los medios estudiados es palpable en unas elecciones que, sin embargo, no tenían un carácter local o autonómico.

El alto porcentaje de artículos encuadrados en Nacional durante la campaña para las elecciones europeas apunta al perfil que suelen adquirir éstas, más centradas en asuntos internos que en temas con una dimensión plenamente europea, algo visible también en la escasa proporción de textos publicados en Internacional (1,5\%). El bajo porcentaje de artículos en Nacional durante la campaña de las generales de 2008 (2,9\%) obedece en buena medida a que los periódicos crearon secciones especiales durante los quince días previos a los comicios. De ahí que la cifra referida a otras secciones del periódico alcance un elevado $22,5 \%$.

Los datos acerca de las unidades de análisis publicadas en portada arrojan algo de luz sobre la importancia otorgada por la prensa aragonesa a cada una de las elecciones consideradas. Mientras que el 6,5\% de los textos de la cobertura de las generales apareció en ese lugar preeminente de los periódicos, únicamente el 3,5\% de los artículos sobre las europeas encontró cabida en primera página.

Tabla 3. Ubicación de la unidad de análisis

\begin{tabular}{|c|c|c|c|}
\hline Elecciones generales 2008 & $\%$ & Elecciones europeas 2009 & $\%$ \\
\hline Regional & 30,1 & Nacional & 35,5 \\
\hline Opinión & 12,7 & Regional & 26,4 \\
\hline Local & 9,9 & Opinión & 19,1 \\
\hline Portada & 6,5 & Local & 12 \\
\hline Internacional & 4,9 & Portada & 3,5 \\
\hline Economía & 4,9 & Internacional & 1,5 \\
\hline Sociedad & 4,3 & Contraportada & 0,7 \\
\hline Nacional & 2,9 & Sociedad & 0,2 \\
\hline Contraportada & 1,2 & Otros & 1,2 \\
\hline Cultura & 0,1 & Total & 100 \\
\hline Otros & 22,5 & & \\
\hline Total & 100 & & \\
\hline
\end{tabular}

Fuente: Elaboración propia

Como resulta de la tabla 4 , no hay diferencias destacables entre ambas coberturas en cuanto a la autoría de las unidades de análisis. Cabe comentar que el porcentaje de artículos procedentes de agencia fue mayor en el caso de las elecciones europeas $(34,7 \%)$ que en el de las generales $(20,5 \%)$, lo que indica la menor importancia concedida por la prensa a las primeras. A la misma conclusión se llega al observar las informaciones escritas por los 
miembros de la redacción de cada periódico: en las generales supusieron el 55,9\% y en las europeas, el $43,1 \%$.

Tabla 4. Autoría de la unidad de análisis

\begin{tabular}{|c|c|c|c|}
\hline Elecciones generales 2008 & $\%$ & Elecciones europeas 2009 & $\%$ \\
\hline Redacción & 55,9 & Redacción & 43,1 \\
\hline Agencia & 20,5 & Agencia & 34,7 \\
\hline Colaborador & 9,5 & Columnista & 13,6 \\
\hline Columnista & 9,3 & Redacción + agencia & 3,3 \\
\hline Humorista gráfico & 1,8 & Colaborador & 3 \\
\hline Redacción + agencia & 1,7 & Humorista gráfico & 2,2 \\
\hline Otros & 1,7 & Total & 100 \\
\hline Total & 100 & & \\
\hline
\end{tabular}

Fuente: Elaboración propia

Los géneros periodísticos de las unidades de análisis no presentan variaciones considerables dependiendo de una u otra cobertura, como muestra la tabla 5. Tanto en 2008 como en 2009 la noticia fue el más empleado: un 70\% aproximadamente. Los géneros de opinión tuvieron una importancia destacada: un 22,7\% de los artículos publicados en la prensa aragonesa durante la campaña de 2008 y un $20 \%$ de los aparecidos durante la campaña de 2009 fueron artículos de opinión, columnas o editoriales.

Tabla 5. Género periodístico de la unidad de análisis

\begin{tabular}{|c|c|c|c|}
\hline Elecciones generales 2008 & $\%$ & Elecciones europeas 2009 & $\%$ \\
\hline Noticia & 69 & Noticia & 71,1 \\
\hline Columna/artículo de opinión & 21,3 & Columna/artículo de opinión & 17,8 \\
\hline Entrevista & 2,8 & Viñeta de humor gráfico & 2,3 \\
\hline Reportaje & 2,2 & Editorial & 2,2 \\
\hline Viñeta de humor gráfico & 1,9 & Entrevista & 2,2 \\
\hline Editorial & 1,4 & Reportaje & 1,3 \\
\hline Otros & 1,4 & Otros & 3,2 \\
\hline Total & 100 & Total & 100 \\
\hline
\end{tabular}

Fuente: Elaboración propia

En cuanto al lugar en el que se originó el hecho sobre el que trata el artículo, en la tabla 6 se ve una presencia similar de las tres provincias aragonesas en las dos coberturas electorales. Zaragoza aglutina en torno al $20 \%$ en ambas, a continuación Teruel recoge algo más del $12 \%$ también en las dos coberturas y, por último, Huesca oscila entre poco más del $8 \%$ en las europeas y casi el $10 \%$ en las generales. 
Al sumar resultados se obtiene que el $42,6 \%$ de las piezas publicadas durante la campaña de 2008 y el 39,4\% de las aparecidas en 2009 tuvieron su origen en Aragón. Por otro lado, se originaron en otras partes de España, con el lógico predominio de Madrid, el 31,3\% de los artículos de 2008 y el 33,6\% de los de 2009.

Tabla 6. Lugar donde se origina el hecho informativo

\begin{tabular}{|c|c|c|c|}
\hline Elecciones generales 2008 & $\%$ & Elecciones europeas 2009 & $\%$ \\
\hline Zaragoza & 20,6 & Otros lugares de España & 21,3 \\
\hline Madrid & 18,4 & Zaragoza & 18,3 \\
\hline Otros lugares de España & 12,9 & Teruel & 12,8 \\
\hline Teruel & 12,1 & Madrid & 12,3 \\
\hline Huesca & 9,9 & Huesca & 8,3 \\
\hline Extranjero & 0,1 & Otro estado UE & 6,1 \\
\hline Sin identificar & 25,9 & Otro estado no UE & 0,3 \\
\hline Total & 100 & Sin identificar & 20,6 \\
\hline & & Total & 100 \\
\hline
\end{tabular}

Fuente: Elaboración propia

El tema dominante en las dos coberturas analizadas fue, como se muestra en la tabla 7, la economía. La crisis, todavía vislumbrada en 2008 , no fue ajena a que el $18,5 \%$ de los artículos se centrase en cuestiones económicas. Durante la campaña de las europeas en 2009 , una crisis ya constatada hizo que el $25,6 \%$ de los textos versara sobre ese asunto, doblando casi el resultado del segundo tema más frecuente en la cobertura.

La segunda posición fue ocupada por temas diferentes en cada una de las elecciones. En 2008 el terrorismo concentró el 9,4\% de los artículos, mientras que en 2009 sólo el 0,3\% de los escritos lo tuvo como tema principal. Estos datos se deben principalmente a la irrupción de ETA en la campaña de las generales con el asesinato el 7 de marzo de Isaías Carrasco, ex concejal socialista en Mondragón. Si bien no directamente, un tema vinculado con el terrorismo surgió al comienzo de la campaña de las europeas: Arnaldo Otegi, dirigente del ilegalizado partido Batasuna, pidió el voto en rueda de prensa para Iniciativa Internacionalista (II), formación que había sido recientemente legalizada por el Tribunal Constitucional pese a las sospechas sobre su naturaleza como pantalla política de ETA. Estas declaraciones provocaron que el debate acerca de la legalización o ilegalización de Iniciativa Internacionalista tuviese una cobertura destacada, aglutinando el 3,2\% de las informaciones publicadas por la prensa aragonesa.

Continuando con la cobertura de las europeas, el segundo y el tercer tema más tratados fueron, respectivamente, la corrupción/escándalos políticos (14\%) y la abstención/euroescepticismo (13\%). Este último asunto siempre está presente en el contexto europeo, pues son habituales las llamadas de los políticos a la participación, sin embargo no 
resulta tan recurrente en el caso de las elecciones generales. La campaña de 2009 estuvo jalonada por el empleo de casos de corrupción y escándalos políticos como tema de campaña, lo que llevó a tan elevado índice de cobertura mediática. La prensa se hizo eco de casos como el "Gürtel" o la utilización del avión oficial para acudir a actos electorales. En el ámbito de la Comunidad Autónoma de Aragón destacó en este sentido la "Operación Molinos", dentro de la cual fueron detenidas varias personas en el municipio zaragozano de La Muela, incluida la alcaldesa de la localidad, acusadas de corrupción urbanística. Por el contrario, estos asuntos no tuvieron presencia significativa en la cobertura de 2008, donde sí la tuvo el agua, tema especialmente sensible en Aragón por el rechazo mayoritario en esta Comunidad al trasvase del Ebro. El 9,2\% de los artículos publicados durante la campaña de las generales tuvo el agua como tema principal, un porcentaje que se redujo a un escaso $0,5 \%$ en 2009.

Tabla 7. Tema principal de la unidad de análisis

\begin{tabular}{|c|c|c|c|}
\hline Elecciones generales 2008 & $\%$ & Elecciones europeas 2009 & $\%$ \\
\hline Economía & 18,5 & Economía & 25,6 \\
\hline Terrorismo & 9,4 & Corrupción/escándalos políticos & 14 \\
\hline Agua & 9,2 & Abstención/euroescepticismo & 13 \\
\hline Estado autonómico & 6,7 & Organización del partido para las elecciones & 7,1 \\
\hline Datos de encuestas & 4,9 & Instituciones UE & 5,3 \\
\hline Inmigración & 2,7 & Estado autonómico & 3,5 \\
\hline Educación & 2 & Ilegalización de II & 3,2 \\
\hline Política exterior & 1,6 & Datos de encuestas & 2,2 \\
\hline Relaciones Iglesia-Estado & 1,5 & Educación & 2,2 \\
\hline Vivienda & 1 & Inmigración & 1,5 \\
\hline Otros & 42,5 & Referencias guerra civil/franquismo & 1,5 \\
\hline Total & 100 & Vivienda & 1,2 \\
\hline & & Relaciones con estados no UE & 1 \\
\hline & & Relaciones con estados UE & 0,7 \\
\hline & & Agua & 0,5 \\
\hline & & Relaciones Iglesia-Estado & 0,5 \\
\hline & & Terrorismo & 0,3 \\
\hline & & Otros & 16,9 \\
\hline & & Total & 100 \\
\hline
\end{tabular}

Fuente: Elaboración propia

Como se observa en la tabla 8, la personalidad política que más artículos protagonizó en ambas coberturas fue el Presidente del Gobierno. Destaca que en la campaña de las generales de 2008, Rodríguez Zapatero protagonizara el 21,1\% de las piezas publicadas por la prensa aragonesa. Su papel como actor principal se redujo, no obstante, en la campaña de las europeas de 2009 al 11,5\%. 
En ambas coberturas el segundo actor político que más textos protagonizó fue Mariano Rajoy, Presidente del Partido Popular y candidato por esta formación a la Presidencia del Gobierno en 2008. Resalta la distancia que separó al líder de la oposición de Rodríguez Zapatero en la campaña de las generales, ya que este último encabezó más del doble de artículos que su oponente político (9,9\%). Por el contrario, Rajoy recortó distancias en 2009 $(10,8 \%)$, quedándose a menos de un punto porcentual del protagonismo periodístico del Jefe del Ejecutivo.

Merece asimismo mención que entre los cinco principales protagonistas de las dos coberturas figurasen políticos nacionales, esto es no aragoneses, de los dos principales partidos políticos españoles y que además no se presentaban como candidatos a las elecciones. Esto indica una presencia considerable en las páginas de los rotativos, durante el periodo de campaña electoral, de políticos con peso en sus partidos pero que no son candidatos.

En el caso de las elecciones generales de 2008, dejando al margen a Rodríguez Zapatero y a Rajoy, los candidatos con un mayor protagonismo en la cobertura fueron los políticos aragoneses que se presentaban a las Cortes por el PSOE (7,2\%) y por el PP (5,8\%), seguidos de cerca por los candidatos del Partido Aragonés $(5,6 \%)$ y de Chunta Aragonesista $(4,8 \%)$.

Durante la campaña de las europeas de 2009, el protagonismo de los candidatos, aragoneses o no, fue más limitado. Los más destacados, con un 6,1\%, fueron los políticos aragoneses en las listas de Europa de los Pueblos-Los Verdes (EP-V), coalición electoral en la que estaba integrada Chunta Aragonesista $(\mathrm{CHA})$. Les siguieron los candidatos aragoneses en la lista de Izquierda Unida, con un 4,2\%. Se observa claramente cómo en el caso de los dos grandes partidos, el protagonismo durante la campaña recayó mayoritariamente sobre los hombros de pesos pesados que no formaban parte de las candidaturas. A este respecto, cabe resaltar que incluso los dos cabezas de lista de PSOE y PP tuvieron un protagonismo limitado en la cobertura: un $4 \%$ en el caso de Juan Fernando López Aguilar y un 2,5\% en el de Jaime Mayor Oreja.

La presencia de terroristas en la cobertura de 2008 se debió, fundamentalmente, al ya comentado asesinato del ex concejal socialista Isaías Carrasco a manos de ETA durante la campaña. Este índice de protagonismo del 1,8\% se redujo a un residual 0,2\% en 2009.

La decisión del Partido Aragonés (PAR) de no presentarse a las elecciones europeas provocó que su notable presencia en las generales de 2008 se tornase en una práctica desaparición $(0,8 \%)$ de las páginas de la prensa aragonesa durante la campaña de 2009. 
Tabla 8. Protagonista principal de la unidad de análisis

\begin{tabular}{|c|c|c|c|}
\hline Elecciones generales 2008 & $\%$ & Elecciones europeas 2009 & $\%$ \\
\hline J. L. Rodríguez Zapatero & 21,1 & J. L. Rodríguez Zapatero & 11,5 \\
\hline Mariano Rajoy & 9,9 & Mariano Rajoy & 10,8 \\
\hline $\begin{array}{l}\text { Candidatos aragoneses del } \\
\text { PSOE }\end{array}$ & 7,2 & Políticos nacionales del PSOE no candidatos & 6,5 \\
\hline $\begin{array}{l}\text { Políticos nacionales del PSOE } \\
\text { no candidatos }\end{array}$ & 6,3 & Candidatos aragoneses de EP-V & 6,1 \\
\hline $\begin{array}{l}\text { Políticos nacionales del PP no } \\
\text { candidatos }\end{array}$ & 6,2 & Políticos nacionales del PP no candidatos & 5,8 \\
\hline Candidatos aragoneses del PP & 5,8 & Instituciones UE & 5,3 \\
\hline Candidatos del PAR & 5,6 & Candidatos aragoneses de IU & 4,2 \\
\hline Otros partidos políticos & 5,4 & J. F. López Aguilar & 4 \\
\hline Candidatos de CHA & 4,8 & Candidatos aragoneses del PSOE & 3,3 \\
\hline Candidatos aragoneses de IU & 3,9 & Políticos no candidatos UE & 3 \\
\hline Gaspar Llamazares & 3,5 & $\begin{array}{c}\text { Políticos aragoneses del PSOE no } \\
\text { candidatos }\end{array}$ & 2,8 \\
\hline Marcelino Iglesias & 2 & Políticos y candidatos de UPyD & 2,8 \\
\hline Medios de comunicación & 2 & Jaime Mayor Oreja & 2,5 \\
\hline Terroristas & 1,8 & Candidatos aragoneses del PP & 2,3 \\
\hline $\begin{array}{l}\text { Políticos aragoneses del PP no } \\
\text { candidatos }\end{array}$ & 1,7 & Políticos aragoneses del PP no candidatos & 2,3 \\
\hline $\begin{array}{c}\text { Políticos aragoneses del PSOE } \\
\text { no candidatos }\end{array}$ & 1,7 & Políticos y candidatos de II & 1,8 \\
\hline Políticos de CHA no candidatos & 0,7 & Candidatos no aragoneses del PSOE & 1,7 \\
\hline Políticos del PAR no candidatos & 0,6 & Marcelino Iglesias & 1,7 \\
\hline $\begin{array}{l}\text { Políticos aragoneses de IU no } \\
\text { candidatos }\end{array}$ & 0,5 & $\begin{array}{l}\text { Políticos y candidatos de Coalición por } \\
\text { Europa }\end{array}$ & 1,7 \\
\hline $\begin{array}{l}\text { Políticos nacionales de IU no } \\
\text { candidatos }\end{array}$ & 0,3 & Willy Meyer & 1,3 \\
\hline Otros & 9,2 & Políticos nacionales de IU no candidatos & 1,3 \\
\hline Total & 100 & $\begin{array}{l}\text { Políticos y candidatos no aragoneses de EP- } \\
\qquad V\end{array}$ & 1.3 \\
\hline & & Políticos aragoneses de IU no candidatos & 1,2 \\
\hline & & Cayo Lara & 1 \\
\hline & & $\begin{array}{c}\text { Políticos no candidatos de CHA } \\
(\text { EP-V) }\end{array}$ & 1 \\
\hline & & Políticos del PAR & 0,8 \\
\hline & & Candidatos no aragoneses del PP & 0,7 \\
\hline & & Medios de comunicación & 0,5 \\
\hline
\end{tabular}

lberoamericana de Comunicación 


\begin{tabular}{|l|c|c|c|}
\hline & & Políticos de estados no UE & 0,5 \\
\hline & & Candidatos de otros estados UE & 0,3 \\
\hline & & Candidatos no aragoneses de IU & 0,2 \\
\hline & & Políticos y candidatos de otros partidos & 0,2 \\
\hline & & Terroristas & 0,2 \\
\hline & & Otros & 9,4 \\
\hline & & Total & 100 \\
\hline
\end{tabular}

Fuente: Elaboración propia

\section{Conclusiones}

Los datos obtenidos confirman la hipótesis planteada al inicio de esta investigación acerca de una cobertura de inferior rango en el caso de los comicios al Parlamento Europeo. Estos resultados refuerzan la conclusión de otros trabajos sobre la menor consideración mediática de las elecciones europeas frente a las generales. El mayor porcentaje de artículos dedicados a la cobertura de estas últimas, así como el menor índice de aparición en portada y una dependencia superior de las agencias de noticias en el caso de la campaña de las europeas apuntan en esta dirección.

En relación con lo anterior, las elecciones europeas adquieren unos rasgos más propios de unas nacionales. Esto se observa en que buena parte de los temas principales de la cobertura de los comicios al Parlamento Europeo son asuntos de interés preferentemente nacional: crisis económica, corrupción política, organización del partido, Estado autonómico, legalización de Iniciativa Internacionalista...

La consideración de las europeas como una especie de ensayo de elecciones nacionales prevalece con los protagonistas de la cobertura. El Presidente del Gobierno y el líder de la oposición son los actores principales, al igual que sucede en las elecciones generales. El peso de figuras políticas no aragonesas tanto del PSOE como del PP que no se presentan como candidatas adquiere en las europeas una notable dimensión, similar a la que adopta en las generales. No ocurre lo mismo con los cabezas de lista de los principales partidos para las elecciones al Parlamento Europeo: López Aguilar y Mayor Oreja quedan relegados, respectivamente, a los puestos octavo y decimotercero en la escala de protagonistas.

Si bien el encuadre nacional es el dominante en ambas coberturas, el aragonés está presente, sobre todo, en cuestiones formales como la ubicación (con un porcentaje muy elevado de piezas en las secciones de Regional y de Local) o el lugar en el que se produce el asunto sobre el que tratan los artículos. La presencia del enfoque europeo es mínima en aspectos formales, pero algo más destacada en cuestiones de contenido como demuestra la presencia de la abstención y el euroescepticismo o las instituciones de la Unión Europea entre los temas principales de la cobertura de 2009. 


\section{Bibliografía}

Asociación de Editores de Diarios Españoles (2010): Libro Blanco de la Prensa Diaria 2010. Madrid: AEDE.

Berganza, M. R. (2009): "Framing the European Union and Building the Media Agenda: The 2004 European Parliamentary Elections in the Spanish Daily Press". Journal of Political Marketing, vol. 8, oㅜ 1, pp. 59-69.

Berganza, M. R. (2008a): "Las elecciones al Parlamento Europeo como comicios de segundo orden. Estudio de la cobertura informativa en televisión (1999-2004)". Estudios sobre el Mensaje Periodístico, no 14, pp. 15-31.

Berganza, M. R. (2008b): "Medios de comunicación, 'espiral del cinismo' y desconfianza política. Estudio de caso de la cobertura mediática de los comicios electorales europeos". ZER, vol. 13, № 25, pp. 121-139.

Canel, M. J. (2006): Comunicación política. Una guía para su estudio y práctica. Madrid: Tecnos.

Canel, M. J., e Innerarity, C. (2000): “La campaña electoral en la televisión”, en Martínez, A., y Méndez, M. (Eds.), Las elecciones al Parlamento Europeo, 1999. Valencia: Tirant lo Blanch, pp. 171-190.

Centro de Investigaciones Sociológicas: Encuesta post-electoral de las elecciones al Parlamento Europeo 2009 [en línea] [Consulta: 6 noviembre 2010]. $<$ http://www.cis.es/cis/opencm/ES/1 encuestas/estudios/ver.jsp?estudio=9802>

De Vreese, C. H. (2009): "Second-Rate Election Campaigning? An Analysis of Campaign Styles in European Parliamentary Elections". Journal of Political Marketing, vol. 8, no 1, pp. 719.

Galindo, F. (2010): "Antecedentes y usos de la investigación en comunicación política en Portugal. De la Revolución de los Claveles al Tratado de Lisboa". DOXA, ํㅜ 10, pp. 57-75.

Kevin, D. (2003): Europe in the Media. A Comparison of Reporting, Representation, and Rhetoric in National Media Systems in Europe. Mahwah: Lawrence Erlbaum Associates.

Maarek, P. (Dir.) (2007): Chronique d'un "non" annoncé: la communication politique et l'Europe (juin 2004-mai 2005). París: L'Harmattan, 2007.

Morata, F. (2000): "El Parlamento Europeo: una institución en busca de su papel político", en Martínez, A., y Méndez, M. (Eds.) (2000). Las elecciones al Parlamento Europeo, 1999. Valencia: Tirant lo Blanch, pp. 13-59.

Picarella, L. (2008): "Las elecciones de 9 de marzo de 2008 en España”. Aposta. Revista de Ciencias Sociales, № 39, pp. 1-21.

Sádaba, T. (2008): Framing: el encuadre de las noticias. El binomio terrorismo-medios. La Crujía: Buenos Aires. 
Vicente Mariño, M., y López Rabadán, P. (2009): "Resultados actuales de la investigación sobre framing: sólido avance internacional y arranque de la especialidad en España". ZER, vol. 14, nำ 26, pp. 13-34.

Zurutuza Muñoz, C., y García Ortega, C. (2012): "Las elecciones europeas de 2009 en la prensa aragonesa”. Revista Latina de Comunicación Social, no 67, pp. 1-22. Disponible en:

http://www.revistalatinacs.org/067/art/945 Zaragoza/01 Cristina.html

\section{Ricardo Zugasti Azagra}

Facultad de Ciencias de la Comunicación

Universidad San Jorge (USJ)

Campus Universitario Villanueva de Gállego

Autovía A-23 Zaragoza-Huesca Km. 299

CP: 50.830, Villanueva de Gállego (Zaragoza)

Tef: +34976060100

Fax. + 34976077584

Email: rzugasti@usj.es

Licenciado en Periodismo y Doctor en Comunicación por la Universidad de Navarra. Acreditado por ANECA como profesor contratado doctor. Ha sido profesor ayudante doctor en la Universidad de Navarra, así como Visiting Research Fellow en la Universidad de Sheffield (Reino Unido) y profesor visitante en la Universidad del Istmo (Guatemala), la Burgas Free University (Bulgaria) y el Instituto Politécnico de Lisboa (Portugal). Su investigación y su docencia están vinculadas a las áreas de Historia de la Comunicación y de Comunicación Política. Pertenece al grupo de investigación "Medios de comunicación y campañas electorales en Aragón", reconocido como grupo emergente por el Gobierno de Aragón.

\section{Carmela García Ortega}

Facultad de Ciencias de la Comunicación

Universidad San Jorge (USJ)

Campus Universitario Villanueva de Gállego

Autovía A-23 Zaragoza-Huesca Km. 299

CP: 50.830, Villanueva de Gállego (Zaragoza)

Tef: +34 976060100

Fax. + 34976077584

Email: cgarcia@usj.es 
Licenciada en Periodismo y Doctora en Comunicación por la Universidad de Navarra. Acreditada por ANECA en la figura de Profesor Ayudante Doctor. En la actualidad desarrolla su actividad docente e investigadora en la Facultad de Ciencias de la Comunicación de la Universidad San Jorge (Zaragoza), donde es Coordinadora del Grado de Periodismo y Coordinadora del Plan de Acción Tutorial. Es la investigadora principal del grupo "Medios de comunicación y campañas electorales en Aragón", reconocido como emergente por el Gobierno de Aragón. Sus áreas de investigación son la comunicación política, la historia del periodismo y el estudio de los métodos de investigación en comunicación. Ha participado en numerosos congresos de ámbito nacional e internacional, ha publicado artículos en revistas científicas y capítulos en obras colectivas y es coautora de varios libros.

\section{Nota}

Este trabajo forma parte de los resultados del grupo de investigación "Medios de comunicación y campañas electorales en Aragón", reconocido como grupo de investigación emergente por el Gobierno de Aragón y financiado por éste (Boletín Oficial de Aragón, no 89, de 9 de mayo de 2011).

\section{Forma de citar este artículo en bibliografías}

ZUGASTI AZAGRA, R. y GARCÍA ORTEGA, C. (2012): "La cobertura en la prensa aragonesa de dos campañas "nacionales": las elecciones europeas de 2009 frente a las generales de 2008", en Revista PANGEA, 3, páginas 23 a 38. Red Académica Iberoamericana de Comunicación. Recuperado el http://revistapangea.org de 2 de: 